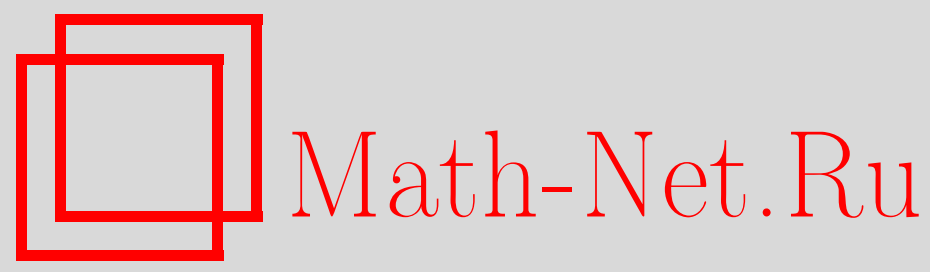

Г. А. Омельянов, Д. А. Кулагин, Асимптотика кинк-кинк взаимодействия для уравнений типа sine-Gordon, Mameм. заметки, 2004, том 75, выпуск 4, 603-607

DOI: https://doi.org/10.4213/mzm55

Использование Общероссийского математического портала Math-Net.Ru подразумевает, что вы прочитали и согласны с пользовательским соглашением http://www.mathnet.ru/rus/agreement

Параметры загрузки:

IP : 54.198 .55 .26

26 апреля 2023 г., $15: 25: 40$ 


\section{АСИМПТОТИКА КИНК-КИНК ВЗАИМОДЕЙСТВИЯ ДЛЯ УРАВНЕНИЙ ТИПА SINE-GORDON}

Д. А. Кулагин, Г. А. Омельянов

Рассматривается класс полулинейных волновых уравнений с малым параметром $\varepsilon$. Нелинейность $F(u)$ предполагается такой, что соответствующее уравнение имеет точное самоподобное решение типа кинка. Для $F(u)$ найдены достаточные условия, при вьполнении которых два кинка взаимодействуют (в смысле главного члена асимптотики по $\varepsilon$ ) по тому же сценарию, что и кинки уравнения sine-Gordon.

Библиографиия: 9 названий.

Рассмотрим полулинейное волновое уравнение с параметром $\varepsilon$

$$
\varepsilon^{2}\left(u_{t t}-u_{x x}\right)+F^{\prime}(u)=0 .
$$

Условия

$$
\begin{aligned}
& F(z) \in C^{\infty}\left(\mathbb{R}^{1}\right), \quad F(z)>0 \text { для } z \in(0,1), \\
& F^{(i)}\left(z_{0}\right)=0, \quad i=0,1, \ldots, K, \quad F^{(K+1)}\left(z_{0}\right)>0 \quad \text { для } z_{0}=0 \quad \text { и } z_{0}=1,
\end{aligned}
$$

где $K=1$ или $K=3$, гарантируют существование самоподобного решения уравнения (1) типа кинка

$$
\begin{array}{rlrl}
u(x, t, \varepsilon) & =\omega\left(\beta \frac{x-V t}{\varepsilon}\right), \quad \beta^{2}=\left(1-V^{2}\right)^{-1}, & \omega(\eta) \in C^{\infty}\left(\mathbb{R}^{1}\right), \\
\omega(\eta) & \rightarrow 0 \quad \text { при } \eta \rightarrow-\infty, \quad \omega(\eta) \rightarrow 1 \quad \text { при } \eta \rightarrow+\infty,
\end{array}
$$

которое стабилизируется на бесконечностях со скоростью не ниже $1 / \eta$. При дополнительном предположении

$$
F\left(\frac{1}{2}+z\right)=F\left(\frac{1}{2}-z\right)
$$

функция $\omega(\eta)-1 / 2$ является нечетной, $\omega(\eta)+\omega(-\eta)=1$. Примерами таких $F$ являются

$$
F(z)=\frac{1-\cos (2 \pi z)}{4 \pi^{2}}, \quad F(z)=\sin ^{4}(\pi z)
$$

Работа выполнена при частичной поддержке фонда CONACYT (Мексика), грант F-41421. 
Первьй пример соответствует уравнению sine-Gordon (уравнение SG). Хорошо известно, что кинки уравнения SG взаимодействуют без изменения своей формы и единственным результатом взаимодействия является появление фазового сдвига [1]. Возникает естественный вопрос: можно ли найти такие условия на $F$, при выполнении которых столкновение кинков уравнения (1) будет происходить по тому же сценарию, что и для уравнения SG (SG-сценарию)?

Точное решение задачи Коши для уравнения (1) неизвестно [2]. Поэтому мы построим асимптотическое решение, трактуя $\varepsilon$ как мальй параметр. Заметим, что кинки (2) стремятся к функции Хевисайда при $\varepsilon \rightarrow 0$. Таким образом, мы должны использовать слабое определение решения, которое позволяет переходить к пределу при $\varepsilon \rightarrow 0$. Однако определение асимптотического по $\bmod O\left(\varepsilon^{2}\right)$ решения, построенного на базе стандартного $\mathscr{D}^{\prime}$-определения, для уравнения (1) не предписьвает для кинков никакого закона движения. Чтобы преодолеть это препятствие, определим асимптотическое решение следующим образом.

ОПРЕДЕЛЕНИЕ 1. Последовательность функций $u(t, x, \varepsilon)$, принадлежащая пространству $C^{\infty}\left(0, T ; C^{\infty}\left(\mathbb{R}_{x}^{1}\right)\right)$ при $\varepsilon>0$ и пространству $C\left(0, T ; \mathscr{D}^{\prime}\left(\mathbb{R}_{x}^{1}\right)\right)$ при $\varepsilon \geqslant 0$, назьвается слабой асимптотикой по $\bmod O_{\mathscr{D}^{\prime}}\left(\varepsilon^{2}\right)$ решения уравнения $(1)$, если для любой тестовой функции $\psi(x) \in \mathscr{D}\left(\mathbb{R}^{1}\right)$ выполняется соотношение

$$
2 \frac{d}{d t} \int_{-\infty}^{\infty} \varepsilon^{2} u_{t} u_{x} \psi d x+\int_{-\infty}^{\infty}\left\{\left(\varepsilon u_{t}\right)^{2}+\left(\varepsilon u_{x}\right)^{2}-2 F(u)\right\} \psi_{x} d x=O\left(\varepsilon^{2}\right)
$$

где правая часть принадлежит $C^{\infty}(0, T)$ при $\varepsilon>0$ и является кусочно-непрерьвной функцией $t$ равномерно по $\varepsilon \geqslant 0$, а оценка понимается как максимум модуля функции $t$.

Левая часть (4) представляет собой результат умножения уравнения $(1)$ на $\psi(x) u_{x}$ и интегрирования по частям в случае гладкой $u$. Таким образом, она равна нулю для любого точного кинк-решения. С другой стороны, соотношение (4) есть условие ортогональности, возникающее для однофазной асимптотики (в случае $F=F(u, t, x, \varepsilon)$ см. [3], [4]). Это условие, во-первьх, гарантирует существование первой поправки, а во-вторых, приводит к уравнению, описывающему движение фронта искаженного кинка. Данное уравнение, так называемое условие типа Гюгонио, трактуется как предел (1) при $\varepsilon \rightarrow 0$.

Рассмотрим два кинка, не взаимодействующих при $t=0$ :

$$
\left.u\right|_{t=0}=\sum_{i=1}^{2} \omega\left(\beta_{i} \frac{x-x_{i}^{0}}{\varepsilon}\right),\left.\quad \varepsilon u_{t}\right|_{t=0}=-\sum_{i=1}^{2} \beta_{i} C_{i} V_{i} \omega^{\prime}\left(\beta_{i} \frac{x-x_{i}^{0}}{\varepsilon}\right),
$$

где $x_{i}^{0}$ - константы такие, что $x_{1}^{0}-x_{2}^{0} \geqslant$ const $>0$ равномерно по $\varepsilon, C_{i}= \pm 1, V_{i} \in(0,1)$. Пусть для определенности $C_{2}=1$. Тогда траектории $x=C_{i} V_{i} t+x_{i}^{0}$ имеют точку пересечения $x=x^{*}$ для $C_{1}=-1$ и любых $V_{i} \in(0,1)$, либо для $C_{1}=1$ и $V_{2}>V_{1}$.

Основной результат работы состоит в следующем. 
ТЕОремА 1. Пусть предположсения (A)-(С) выполнены. Пусть также $F$ и $\beta_{i}$ таковы, что

$$
\begin{aligned}
& F(z+1)=F(z) \\
& \int_{-\infty}^{\infty} F(\omega(\eta)+\omega(\theta \eta)) d \eta \leqslant \int_{-\infty}^{\infty}\{F(\omega(\eta))+F(\omega(\theta \eta)) \\
& \left.+2 \beta_{1} \beta_{2} \sqrt{F(\omega(\eta)) F(\omega(\theta \eta))}\right\} d \eta, \quad \theta=\frac{\beta_{1}}{\beta_{2}}
\end{aligned}
$$

Тогда кинки в задаче (1), (5) взаимодействуют по SG-сценарию.

Для обоих примеров (3) все предположения теоремы выполнены.

СХЕМА ДОКАЗАТЕЛЬСТВА ТЕОРЕМЫ. Наметим основные этапы доказательства теоремы. Следуя методу слабых асимптотик [5]-[9], полагаем

$$
u(t, x, \varepsilon)=\sum_{i=1}^{2} \omega\left(\beta_{i} \frac{x-\Phi_{i}(t, \tau, \varepsilon)}{\varepsilon}\right)+O_{\mathscr{D}^{\prime}}(\varepsilon)
$$

где $\Phi_{i}=\varphi_{i 0}(t)+\varepsilon \varphi_{i 1}(\tau), \varphi_{i 0}=C_{i} V_{i} t+x_{i}^{0}, \tau=\beta_{1}\left(\varphi_{20}-\varphi_{10}\right) / \varepsilon, \varphi_{i 1}(\tau) \in C^{\infty}$, $\varphi_{i 1} \rightarrow 0$ при $\tau \rightarrow-\infty$. Момент "времени" $\tau=0$ соответствует пересечению траекторий $x=\varphi_{i 0}(t), i=1,2$, в точке $x=x^{*}$. Поведение $\varphi_{i 1}(\tau)$ при $\tau \rightarrow \infty$ описывает сценарии взаимодействия кинков.

Символом $O_{\mathscr{D}^{\prime}}\left(\varepsilon^{k}\right)$ обозначаются функции $f(x, t, \varepsilon)$, принадлежащие $C^{\infty}(0, T$; $\left.C^{\infty}\left(\mathbb{R}_{x}^{1}\right)\right)$ при $\varepsilon>0$ и принадлежащие $C\left(0, T ; \mathscr{D}^{\prime}\left(\mathbb{R}_{x}^{1}\right)\right)$ при $\varepsilon \geqslant 0$, такие, что равномерно по $\varepsilon \geqslant 0$ для любой $\psi \in \mathscr{D}\left(\mathbb{R}_{x}^{1}\right)$ справедливо соотношение

$$
\int_{-\infty}^{\infty} f(x, t, \varepsilon) \psi(x) d x=O\left(\varepsilon^{k}\right)
$$

где оценка понимается в том же смысле, что и в соотношении (4).

Далее, находим слабую асимптотику выражений, входящих в (4), и убеждаемся, что левая часть этого соотношения есть линейная комбинация $\delta\left(x-x^{*}\right), \varepsilon \delta^{\prime}\left(x-x^{*}\right)$ и $\varepsilon \delta^{\prime}\left(x-\Phi_{i}\right), i=1,2$. Приравнивая к нулю коэффициенты при дельта-функциях, получаем равенство $\beta_{i}^{2}=1 /\left(1-V_{i}^{2}\right)$ и уравнение второго порядка для функции $\sigma=\sigma(\tau)$. Вводя вспомогательную функцию $W$, переписьваем его в виде условий типа Гюгонио

$$
\frac{d \sigma}{d \tau}=P, \quad \frac{d W}{d \tau}=Q .
$$

Здесь

$$
\begin{gathered}
P=\frac{1}{L}\left\{\frac{W}{\mathscr{F}}+\sigma-2\left(b_{1} \bar{\lambda}_{2}+\theta b_{2} \lambda_{2}\right)\right\}, \\
Q=\frac{\mathscr{F}}{2}\left\{\left(1-\theta \lambda_{1}^{2}\right) P^{2}-2\left(1+\lambda_{1}\left(b_{2}-\theta b_{1}\right)\right) P+1+2 \lambda_{1}\left(b_{2}^{2}+\theta b_{1}^{2}\right)\right\}+\frac{1}{\nu^{2}}\left(\lambda_{1}-\frac{B_{\Delta}}{\beta_{1} \beta_{2}}\right),
\end{gathered}
$$


и

$$
\begin{gathered}
L=\sigma+\bar{\lambda}_{2}-\theta \lambda_{2}, \quad \mathscr{F}=\left(1+\theta\left(1+2 \lambda_{1}\right)\right)^{-1}, \\
\sigma=\beta_{1}\left(\Phi_{2}-\Phi_{1}\right) / \varepsilon, \quad \nu=C_{2} V_{2}-C_{1} V_{1}, \\
\lambda_{1}=\frac{1}{a_{2}} \int_{-\infty}^{\infty} \omega^{\prime}(\eta) \omega^{\prime}(\theta \eta+\sigma) d \eta, \quad \lambda_{2}=\frac{1}{a_{2}} \int_{-\infty}^{\infty} \eta \omega^{\prime}(\eta) \omega^{\prime}(\theta \eta+\sigma) d \eta \\
B_{\Delta}=\frac{1}{a_{2}} \int_{-\infty}^{\infty}\{F(\omega(\eta)+\omega(\theta \eta+\sigma))-F(\omega(\eta))-F(\omega(\theta \eta+\sigma))\} d \eta, \\
\bar{\lambda}_{2}=\frac{1}{a_{2}} \int_{-\infty}^{\infty} \eta \omega^{\prime}(\eta) \omega^{\prime}\left(\frac{\eta-\sigma}{\theta}\right) d \eta, \quad a_{2}=\int_{-\infty}^{\infty}\left(\omega^{\prime}(\eta)\right)^{2} d \eta, \quad b_{i}=\frac{C_{i} V_{i}}{\nu} .
\end{gathered}
$$

Отметим, что условия (A)-(D) обеспечивают существование сверток $\lambda_{i}, \bar{\lambda}_{2}, B_{\Delta}$, a также стремление их к нулю при $\sigma \rightarrow \pm \infty$.

Взаимосвязь между функциями $\varphi_{11}, \varphi_{21}$ и $\sigma$ задается уравнениями

$$
\beta_{1} \frac{d \varphi_{11}}{d \tau}=-\mathscr{F} G_{1}, \quad \beta_{2} \frac{d \varphi_{21}}{d \tau}=\theta \mathscr{F} G_{2},
$$

где

$$
G_{1}=\left(1+\theta \lambda_{1}\right) \frac{d \sigma}{d \tau}+2 \theta b_{1} \lambda_{1}-1, \quad G_{2}=\left(1+\lambda_{1}\right) \frac{d \sigma}{d \tau}-2 b_{2} \lambda_{1}-1 .
$$

Динамическая система (7) имеет особенность при $\sigma=0$ и $W \neq 0$. Однако условия (A)-(Е) гарантируют существование сепаратриссы, которая при всех $\tau \in \mathbb{R}^{1}$ лежит в полосе $\left\{\sigma \in \mathbb{R}^{1},|W| \leqslant\right.$ const $\}$ на плоскости $(\sigma, W)$, проходит через точку $(0,0)$ и $\sigma$-координата которой стремится к $\pm \infty$, а $W \rightarrow \pm W^{0}$ при $\tau \rightarrow \pm \infty$. Таким образом, дополняя систему (7) “начальными” условиями

$$
\sigma \rightarrow \tau, \quad W \rightarrow W^{0} \quad \text { при } \tau \rightarrow-\infty,
$$

мы фиксируем траекторию, проходящую из левой полуплоскости в правую.

Заключительный этап доказательства теоремы состоит в установлении того факта, что величина $W^{0}$ меняется при возмущении главного члена асимптотики (6) малыми (в смысле $\left.\mathscr{D}^{\prime}\left(\mathbb{R}_{x}^{1}\right)\right)$ добавками. Например, можно положить

$$
u(t, x, \varepsilon)=\sum_{i=1}^{2}\left(\omega\left(\bar{\beta}_{i} \frac{x-\Phi_{i}(t, \tau, \varepsilon)}{\varepsilon}\right)+A_{i}(\sigma) U\left(\frac{\bar{\beta}_{i}}{\mu_{i}} \frac{x-\Phi_{i}(t, \tau, \varepsilon)}{\varepsilon}\right)\right) .
$$

Здесь $\bar{\beta}_{i}=\beta_{i}+\beta_{i 1}(\tau)$, где $\beta_{i}$ - те же числа, что и раньше, $\beta_{i 1}(\tau) \in C^{\infty}\left(\mathbb{R}^{1}\right)$ - некоторые функции, экспоненциально быстро убьвающие при $\tau \rightarrow \pm \infty$ и такие, что $\bar{\beta}_{i}>0$,

$$
U\left(\frac{\bar{\beta}_{i}}{\mu_{i}} \frac{x-\Phi_{i}(t, \tau, \varepsilon)}{\varepsilon}\right)=\omega^{(2 l+1)}\left(\frac{\bar{\beta}_{i}}{\mu_{i}} \frac{x-\Phi_{i}(t, \tau, \varepsilon)}{\varepsilon}\right)=O_{\mathscr{D}^{\prime}}\left(\left(\varepsilon \mu_{i}\right)^{2 l+1}\right), \quad l \geqslant 1,
$$

$A_{i}(\sigma) \in C^{\infty}$ - нечетные функции, достаточно быстро стремящиеся к нулю при $\sigma \rightarrow \pm \infty$, причем $\left|A_{1}\right|<\left|A_{2}\right|, \mu_{1}$ и $\mu_{2}=\mu_{1} / \theta$ - достаточно малые параметры. Выбирая соответствующие $\beta_{i 1}, A_{i}(\sigma), \mu_{i}$, получим, что $W^{0}=0$. Тогда функции $\varphi_{i 1}(\tau)$, определяемые из уравнений, аналогичных (8), будут ограниченными равномерно по $\tau \in \mathbb{R}^{1}$. Отсюда следует утверждение теоремы 1. 
Теорема 2. Следующие два утверждения әквивалентны.

1. Функиия вида (6) является слабой асимптотикой решения уравнения (1) в смысле определения 1.

2. Функиия вида (6) удовлетворяет закону сохранения

$$
\frac{d}{d t} \int_{-\infty}^{\infty} u_{t} u_{x} d x=0
$$

и әнергетическому соотночению

$$
\frac{d}{d t} \int_{-\infty}^{\infty} x \varepsilon^{2} u_{t} u_{x} d x+\frac{1}{2} \int_{-\infty}^{\infty}\left\{\left(\varepsilon u_{t}\right)^{2}+\left(\varepsilon u_{x}\right)^{2}-2 F(u)\right\} d x=0
$$

отвечающим уравнению (1).

ЗАмЕЧАниЕ 1. Если кинк $u(t, x, \varepsilon)=\omega(\beta(x-C V t) / \varepsilon)$ есть решение уравнения (1), то антикинк $u(t, x, \varepsilon)=\omega(-\beta(x-C V t) / \varepsilon)$ также является его решением. Утверждения теорем верны также и для взаимодействия антикинк-антикинк. Вместе с тем, описание взаимодействия кинк-антикинк оказьвается более сложным. Оно будет рассмотрено отдельно.

\section{СПИСОК ЦИТИРОВАННОЙ ЛИТЕРАТУРЫ}

[1] Захаров В. Е., Тахтаджан Л. А., Фаддеев Л. Д. Полное описание решений уравнения sine-Gordon // Докл. АН СССР. 1974. Т. 219. С. 1334-1337.

[2] ЖКибер А. В., Соколов В. В. Точно интегрируемые гиперболические уравнения лиувиллевского типа // УМН. 2001. Т. 56. №1. С. 63-104.

[3] Maslov V.P., Omel'yanov G. A. Geometric Asymptotics for Nonlinear PDE, I. Providence, RI: Amer. Math. Soc., 2001.

[4] Danilov V.G., Omel'yanov G. A., Radkevich E. V. Hugoniot-type conditions and weak solutions to the phase field system // Europ. J. Appl. Math. 1999. V. 10. P. 55-77.

[5] Маслов В. П. Три алгебры, соответствующие негладким решениям систем квазилинейных гиперболических уравнений // УМН. 1980. Т. 35. № 2. С. 252-253.

[6] Данилов В.Г., Маслов В.П., Шелкович В. М. Алгебры особенностей сингулярных решений квазилинейных строго гиперболических систем первого порядка // ТМФ. 1998. T. 114. №1. C. 3-55.

[7] Danilov V.G., Shelkovich V. M. Propagation and interaction of shock waves of quasilinear equation // Nonlinear Studies. 2001. V. 8. P. 135-170.

[8] Danilov V.G. Generalized solutions describing singularity interaction // Intern. J. Math. Math. Sci. 2002. V. 30. P. 1-14.

[9] Danilov V.G., Omel'yanov G.A., Shelkovich V.M. Weak asymptotics method and interaction of nonlinear waves // Asymptotic Methods for Wave and Quantum Problems (M. V. Karasev, ed.), Amer. Math. Soc. Transl. Ser. 2. V. 208. Providence, RI: Amer. Math. Soc., 2003. P. 33-164.

Московский государственный институт электроники и математики

Поступило

E-mail : kulaginda@mtu-net.ru

25.12.2002 